\title{
RIGOROUS PHOTOGRAMMETRIC PROCESSING OF CHANG'E-1 AND CHANG'E-2 STEREO IMAGERY FOR LUNAR TOPOGRAPHIC MAPPING
}

\author{
Kaichang Di*, Yiliang Liu, Bin Liu, Man Peng \\ Institute of Remote Sensing Applications, Chinese Academy of Sciences \\ P. O. Box 9718, Datun Rd, Chaoyang District, Beijing 100101, P.R.China \\ (kcdi, ylliu, liub, pengman)@irsa.ac.cn
}

Commission IV, WG IV/7

KEY WORDS: Planetary, Topographic Mapping, Rigorous Sensor Model, Chang'E-1, Chang'E-2, CCD image, Model Refinement

\begin{abstract}
:
Chang'E-1(CE-1) and Chang'E-2(CE-2) are the two lunar orbiters of China's lunar exploration program. Topographic mapping using CE-1 and CE-2 images is of great importance for scientific research as well as for preparation of landing and surface operation of Chang'E-3 lunar rover. In this research, we developed rigorous sensor models of CE-1 and CE-2 CCD cameras based on push-broom imaging principle with interior and exterior orientation parameters. Based on the rigorous sensor model, the 3D coordinate of a ground point in lunar body-fixed (LBF) coordinate system can be calculated by space intersection from the image coordinates of conjugate points in stereo images, and the image coordinates can be calculated from 3D coordinates by back-projection. Due to uncertainties of the orbit and the camera, the back-projected image points are different from the measured points. In order to reduce these inconsistencies and improve precision, we proposed two methods to refine the rigorous sensor model: 1) refining EOPs by correcting the attitude angle bias, 2) refining the interior orientation model by calibration of the relative position of the two linear CCD arrays. Experimental results show that the mean back-projection residuals of CE-1 images are reduced to better than 1/100 pixel by method 1 and the mean back-projection residuals of CE-2 images are reduced from over 20 pixels to 0.02 pixel by method 2. Consequently, high precision DEM (Digital Elevation Model) and DOM (Digital Ortho Map) are automatically generated.
\end{abstract}

\section{INTRODUCTION}

Chinese Lunar Exploration Program (CLEP), usually known as the Chang'E program is a program of robotic and human missions to the Moon undertaken by the China National Space Administration (CNSA) (Wikipedia, 2012). Chang'E-1(CE-1) and Chang'E-2(CE-2) are the two lunar probes in phase I of CLEP which is called orbital mission.

CE-1 orbiter is the first lunar probe of China launched on October 24, 2007. It carries a three-line push-broom CCD camera which has a ground resolution of $120 \mathrm{~m}$ and a swath width of $60 \mathrm{~km}$ at $200 \mathrm{~km}$ orbit altitude. It is implemented on an area array CCD sensor and uses only the 11th, 512th and 1013th rows to generate the forward-, nadir-, and backward-looking images simultaneously in the flight direction (Li et al., 2010; Peng et al., 2010).

CE-2 orbiter, launched on October 1, 2010, is the follow-up orbiter of CE-1. As a mission highlight, a local image map at Sinus Iridum, the pre-selected landing site of Chang'E-3 lunar rover produced using CE-2 CCD images was released on November 8, 2010(NAOC, 2010). CE-2 was broadly similar to the CE-1 mission, but had important differences. CE-2 flew at two kinds of orbit altitude, $100 \times 100 \mathrm{~km}$ circular orbit at which the CCD camera can reach a resolution of $7 \mathrm{~m}$ and $100 \times 15 \mathrm{~km}$ elliptical orbit with a resolution of $1.5 \mathrm{~m}$ around the perilune. The former kind of orbit provides high resolution global image coverage of the Moon for various scientific researches, while the latter mainly provides more detailed information for Chang'E-3 lunar landing and surface operation (Zhao et al., 2010b).

* Corresponding author
CE-2 CCD stereo camera adopts a stereo imaging solution with the single lens and two angles of view in the same track and a self-push-broom imaging mode with high sensitivity time delay integration (TDI) CCD (Zhao et al., 2010a). Comparing with the three-line array mode of CE-1 CCD camera, CE-2 adopts a two-line push-broom camera to reduce the pressure of mass data, and meanwhile, assures stereo imaging condition (Zhao et al., 2010b). It can acquire forward- and backward-looking images simultaneously to consist stereo pairs.

At present, a global image mosaic of the Moon using CE-1 CCD images and laser altimeter (LAM) data has been produced (Li et al., 2010). A global image map of the Moon with a resolution of $7 \mathrm{~m}$ has also been released by the CLEP on February 6, 2012 (Xinhua News Agency, 2012). But there's no CE-2 topographic products (e.g., DEM and DOM) released so far. In our previous research, we developed a rigorous sensor model for CE-1 stereo imagery has (Peng et al., 2010) and methods of automatic DEM and DOM generation, and co-registration of CE-1 CCD images and LAM data (Di et al., 2010).

In this paper, we elaborate the rigorous sensor model of CE-2 CCD camera. Firstly, interior orientation model of CE-1 and CE-2 CCD cameras including parameters and structure of the camera are introduced in detail. Secondly, the formulas of exterior orientation model are given to constitute a rigorous geometric model for both CE- 1 and CE-2 cameras. After analyzing the distribution patterns of back-projection residuals, we propose two methods to refine the sensor model: 1) refining EOPs by correcting the attitude angle bias, 2) refining the interior orientation model by calibration of the relative position of the two linear CCD arrays. Finally, experimental results with CE-1 and 
CE-2 images are presented to demonstrate the effectiveness of the proposed methods.

\section{RIGOROUS GEOMETRIC MODEL OF CE-1 AND CE-2 CCD IMAGES}

\subsection{Interior Orientation}

We have developed the interior orientation model of CE-1 CCD camera to calculate focal plane coordinates from pixel coordinates using Equation (1) (Peng et al., 2010).

$$
\begin{aligned}
& x=\left(x_{p}-c c d_{-} \text {line }\right) \cdot \text { pixsize }-x_{0} \\
& y=\left(y_{p}-c o l\right) \cdot \text { pixsize }-y_{0}
\end{aligned}
$$

where pixsize stands for the pixel size of the CCD array; col is the pixel position in column direction; $\left(x_{p}, y_{p}\right)$ are the center position (511.5, 255.5) of the actual imaging area (1024 rows by 512 columns); $\left(x_{0}, y_{0}\right)$ represent principal point position in the focal plane frame; $(x, y)$ is the focal plane coordinate of forward-, nadir-, or backward-looking images. ccd_line is 11, 512, and 1013 for forward-, nadir-, and backward-looking images respectively.

Unlike the CE-1 CCD camera implemented on an area array CCD sensor, the CCD camera (shown in Figure 1) carried by CE-2 is a two-line push-broom sensor assembled on a focal plane separately. The two CCD arrays share the same optical axis with a focal length of $144.3 \mathrm{~mm}$. Each CCD line array has 6,144 pixels. The primary technical parameters are listed in Table 1 (Zhao et al., 2010b).

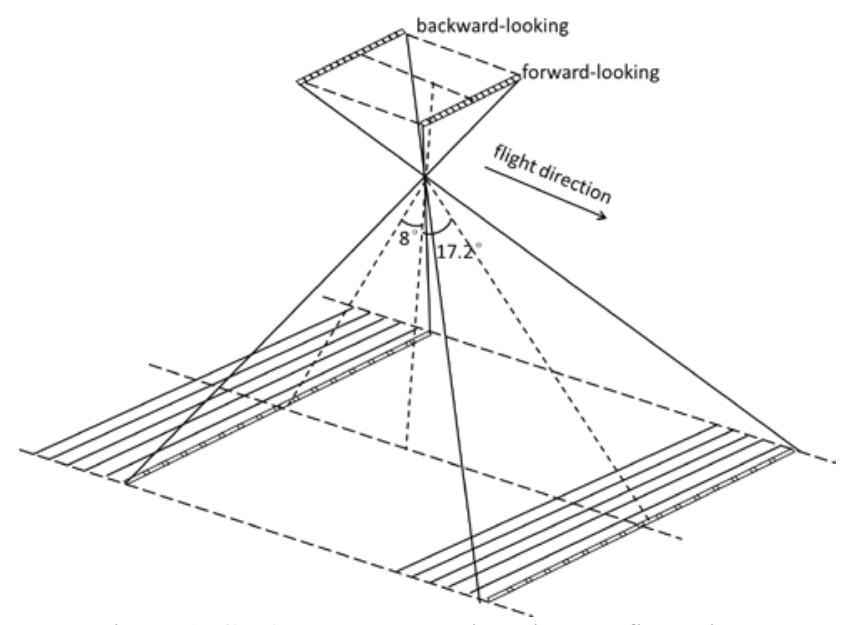

Figure 1. CE-2 stereo camera imaging configuration

\begin{tabular}{|l|c|c|}
\hline Index & $\begin{array}{c}100 \times 100 \mathrm{~km} \\
\text { Circular orbit }\end{array}$ & $\begin{array}{c}100 \times 15 \mathrm{~km} \\
\text { elliptical orbit }\end{array}$ \\
\hline Nominal swath & $43 \mathrm{~km}$ & $6.45 \mathrm{~km}$ \\
\hline Nominal resolution & $7 \mathrm{~m}$ & $1.05 \mathrm{~m}$ \\
\hline Viewing angle & Forward $+8^{\circ} ;$ backward $-17.2^{\circ}$ \\
\hline
\end{tabular}

Table 1. Technical parameters of CE-2 CCD camera

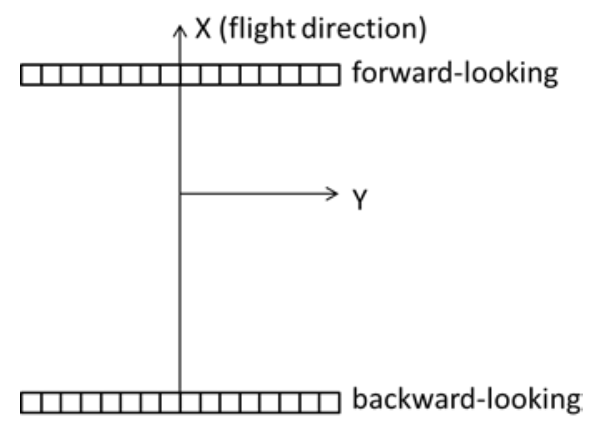

Figure 2. Focal plane of CE-2 CCD camera

According to the focal plane configuration of CE-2 CCD camera shown in Figure 2, we can derive the following interior orientation equation to calculate focal plane coordinates from pixel coordinates.

$$
\begin{aligned}
& x=x_{0}-\tan (\theta) \cdot f \\
& y=y_{0}-\left(\operatorname{col}-s_{0}\right) \cdot \text { pixsize }
\end{aligned}
$$

where $\theta$ represents the viewing angle, which is $8^{\circ}$ for forward-looking images and $-17.2^{\circ}$ for backward-looking images; $f$ is the focal length; col is the column position of an image point; $S_{0}$ is the center of CCD, pixsize stands for the pixel size of the CCD array, which is $10.1 \mu \mathrm{m}$ (Zhao et al., 2010b); ( $\left.x_{0}, y_{0}\right)$ represents the principal point position in the focal plane frame; $(x, y)$ is the focal plane coordinates of forward- or backwardlooking images.

\subsection{Exterior Orientation}

For push-broom sensor, exterior orientation parameters (EOPs) are interpolated from original telemetry data for each scan line. Lagrange polynomials are used in the interpolation (Liu and Di, 2011).

Given the interior orientation model and EOPs after interpolation, we developed the rigorous sensor model of CE-1 and CE-2 CCD imagery in the form of collinearity equation:

$$
\left[\begin{array}{l}
X-X_{s} \\
Y-Y_{s} \\
Z-Z_{s}
\end{array}\right]=\lambda \cdot R_{o l} \cdot R_{b o} \cdot R_{i b} \cdot\left[\begin{array}{l}
X \\
y \\
-f
\end{array}\right]
$$

where $(x, y)$ are focal plane coordinates of an image point; $f$ is the focal length; $(X, Y, Z)$ and $(X s, Y s, Z s)$ are the 3D ground coordinate and the camera center position in lunar body-fixed (LBF) coordinate system; $R_{i b}$ is the rotation matrix from image space coordinate system to spacecraft body coordinate system (BCS); $R_{b o}$ is the rotation matrix from BCS to orbit coordinate systems (OCS); $R_{o l}$ is the rotation matrix from OCS to LBF, $\lambda$ is a scale factor.

In Equation (3), (Xs, Ys, Zs) for each scan line are obtained from spacecraft ephemeris and interpolation; $R_{i b}$ and $R_{b o}$ are obtained from telemetry data. 


\subsection{Space Intersection and Back-projection}

Stereo pairs can be formed by CE-1 and CE-2 images of different looking angles. Based on the rigorous sensor model, the 3D coordinate of a ground point in LBF can be calculated by space intersection from the image coordinates of the conjugate points in stereo images, and the image coordinate can be calculated from 3D coordinate by back-projection. Ideally, using the 3D coordinate from space intersection, the back-projected image positions should be the same as the measured image points which are used in space intersection. However, due to the orbit uncertainty and interior orientation uncertainty, the backprojected image points are different from the measured point. The differences are called back-projection residuals. Some regular patterns usually appear in these residuals, which are extremely useful for analyzing and finding out the error sources and eliminating the inconsistencies.

\section{SENSOR MODEL REFINEMENTS}

In order to reduce the inconsistencies (back-projection residuals) of stereo images and improve mapping precision, we propose two methods to refine the rigorous sensor model: 1) refining EOPs by correcting the attitude angle bias, 2) refining the interior orientation model by calibration of the relative position of the two linear CCD arrays.

\subsection{Refinement of EOPs}

The internal structure of CE-1 stereo camera is very stable because it is implemented in one area array sensor. Thus, the back-projection residuals of CE-1 images are mainly caused by errors of EOPs. For satellite images with high altitude, the position parameters and the attitude parameters have strong correlation. So correcting attitude angle bias can also compensate errors caused by position parameters.

For each image, Equation (3) can be rewritten into Equation (4), and further simplified as Equation (5).

$$
\begin{aligned}
& {\left[\begin{array}{l}
X-X_{S} \\
Y-Y_{s} \\
Z-Z_{s}
\end{array}\right]=\lambda \cdot R \cdot\left[\begin{array}{r}
x \\
y \\
-f
\end{array}\right]} \\
& \frac{u_{2}}{\left|u_{2}\right|}=R \cdot \frac{u_{1}}{\left|u_{1}\right|} \\
& u_{1}=\left[\begin{array}{ll}
x & y-f
\end{array}\right]^{T} \\
& \left|u_{1}\right|=\sqrt{x^{2}+y^{2}+(-f)^{2}} \\
& u_{2}=\left[X-X_{s} Y-Y_{s} Z-Z_{s}\right]^{T} \\
& \left|u_{2}\right|=\sqrt{\left(X-X_{s}\right)^{2}+(Y-Y)^{2}+\left(Z-Z_{s}\right)^{2}}
\end{aligned}
$$

where $R$ is the rotation matrix from image space to LBF. Then we get the partial derivatives $\partial R / \partial \varphi, \partial R / \partial \omega, \partial R / \partial \kappa$ in the form of $3 \times 3$ matrix.

The observation equation for attitude angle bias correction can be represented as Equation (6) (Yuan and Yu, 2008).

$$
v=A \cdot x-L
$$

$$
\text { where } \begin{aligned}
A & =\left[\frac{\partial R}{\partial \varphi} \cdot u_{1}^{\prime} \frac{\partial R}{\partial \omega} \cdot u_{1}^{\prime} \frac{\partial R}{\partial \kappa} \cdot u_{1}^{\prime}\right] \\
L & =-R \cdot u_{1}^{\prime}+u_{2}^{\prime} \\
x & =\left[d \varphi^{\prime} d \omega^{\prime} d \kappa^{\prime}\right]^{T} \\
u_{i}^{\prime} & =\frac{u_{i}}{\left|u_{i}\right|}(i=1,2)
\end{aligned}
$$

Given a set of initial values of attitude angle biases as $d \varphi=0, d \omega=0$ and $d \kappa=0$, rotation matrix $R$ can be calculated using Euler angles $\varphi+d \varphi, \omega+d \omega$, and $\kappa+d \kappa$. In each iteration, the correction values are added to the bias values as shown in Equation (7).

$$
\begin{aligned}
& d \varphi \leftarrow d \varphi+d \varphi^{\prime} \\
& d \omega \leftarrow d \omega+d \omega^{\prime} \\
& d \kappa \leftarrow d \kappa+d \kappa^{\prime}
\end{aligned}
$$

Iteration stops when the correction values are less than a predefined threshold. And finally the attitude angle biases can be figured out.

\subsection{Refinement on Interior Orientation}

Because the two linear CCD arrays are separately assembled on the focal plane of the CE-2 CCD camera, their relative position may be changed in orbit, which can cause significant errors of the sensor model. As a result, back-projection residuals appear to have a regular pattern in image space. These residuals can be used to refine the interior orientation by calibration of the relative position of the two linear CCD arrays.

Assuming the position of forward-looking CCD array and the principal point are fixed, the relationship between the actual and the theoretical positions of backward-looking CCD array can be modeled by scale and translation along the array direction on the focal plane. So the interior orientation formula for backward-looking images in Equation (2) can be rewritten into Equation (8).

$$
y=y_{0}-\left(\mathrm{col}-s_{0}\right) \cdot \text { pixsize } \cdot(1+\text { scale })+\text { offset }
$$

where scale and offset are the scale and translation parameters for the calibration of the backward-looking CCD array. The problem is how to determine the value of scale and offset. According to the residuals distribution, we can fit a least-squares line (LSL) to describe the error trend so that to estimate the two parameters. Let $\mathrm{col}_{k}$ and $r_{k}$ are the column position and residual of point $k$, a line function can be represented as Equation (9). By minimizing the sum of the squares of the $r_{k}$, the two parameters $a$ and $b$ can be estimated.

$$
r_{k}=a \cdot\left(\mathrm{col}_{k}-s_{0}\right)+b
$$

The estimated coefficients $a$ and $b$ of the LSL for both forwardand backward-looking images are summed up as the scale and offset values for the backward-looking CCD array, since we assume the position of forward-looking CCD array and the principal point are fixed. 


\section{EXPERIMENTAL RESULTS}

\subsection{Experiment Using CE-1 CCD Images}

Considering that Sinus Iridum is the pre-selected landing site of Chang'E-3 lunar rover mission, in our experiment, we choose 3 orbits (No.0561, No.0562 and No.0563) of CE-1 CCD images located at Sinus Iridum. Figure 3 shows the partial images (1,000 rows, after grayscale stretching) from three view angles in No.0562 orbit.
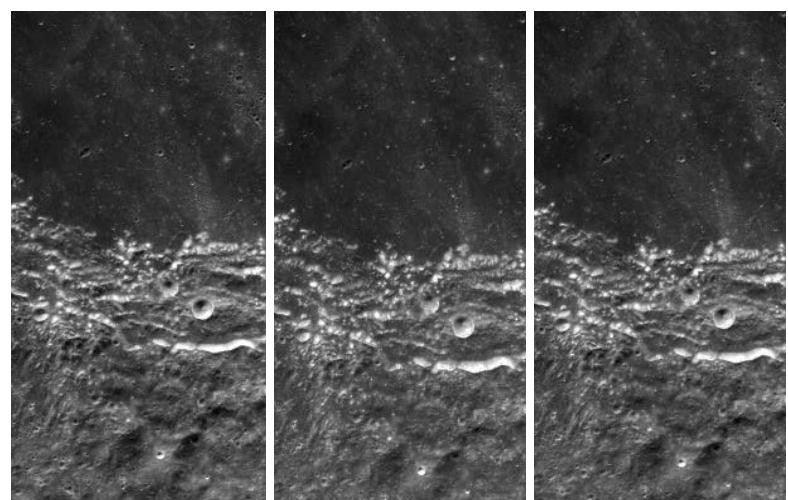

(a) Forward-looking (b) Nadir-looking (c) Backward-looking Figure 3. Partial forward-, nadir-, and backward-looking CCD images taken by CE-1 from No.0562 orbit (120m resolution)

In this experiment, we use 1,000 rows of the forward-, nadir-, backward-looking CCD images of these three orbits. Conjugate points of stereo images are obtained through feature point extraction by SIFT operator (Lowe, 1999) and feature point matching by cross-correlation. Statistics of back-projection residuals of more than 15,000 matched feature points for each image is shown in Table 2. The attitude angle bias shown in Table 3 for each image of these three orbits are added to the EOPs, then ground points are back-projected to image space again and back-projection residuals are recalculated in Table 4.

\begin{tabular}{|c|c|c|c|c|c|}
\hline \multirow{2}{*}{$\begin{array}{c}\text { Orbit } \\
\text { No. }\end{array}$} & \multirow{2}{*}{ Image } & \multicolumn{2}{|c|}{ Column direction } & \multicolumn{2}{c|}{ Row direction } \\
\cline { 3 - 6 } & & $\begin{array}{c}\text { Mean } \\
\text { (pixel) }\end{array}$ & $\begin{array}{c}\text { RMS } \\
\text { (pixel) }\end{array}$ & $\begin{array}{c}\text { Mean } \\
\text { (pixel) }\end{array}$ & $\begin{array}{c}\text { RMS } \\
\text { (pixel) }\end{array}$ \\
\hline \multirow{3}{*}{0561} & $\mathrm{~F}$ & -1.07 & 0.12 & 0.00 & 0.00 \\
\cline { 2 - 6 } & $\mathrm{N}$ & -0.05 & 0.20 & 0.00 & 0.00 \\
\cline { 2 - 6 } & $\mathrm{B}$ & 1.13 & 0.18 & 0.00 & 0.00 \\
\hline \multirow{3}{*}{0562} & $\mathrm{~F}$ & -1.51 & 0.13 & 0.00 & 0.00 \\
\cline { 2 - 6 } & $\mathrm{N}$ & 0.06 & 0.21 & 0.00 & 0.00 \\
\cline { 2 - 6 } & $\mathrm{B}$ & 1.45 & 0.16 & 0.00 & 0.00 \\
\hline \multirow{3}{*}{0563} & $\mathrm{~F}$ & -1.40 & 0.16 & 0.00 & 0.00 \\
\cline { 2 - 6 } & $\mathrm{N}$ & 0.02 & 0.26 & 0.00 & 0.00 \\
\cline { 2 - 6 } & $\mathrm{B}$ & 1.38 & 0.19 & 0.00 & 0.00 \\
\hline
\end{tabular}

Table 2. Back-projection residuals for CE-1 CCD images before correction (F, N and B are forward-, nadir- and backward- looking for short, the same below)

\begin{tabular}{|c|c|c|c|c|}
\hline $\begin{array}{c}\text { Orbit } \\
\text { No. }\end{array}$ & Image & $\phi$ (degree) & $\omega$ (degree) & $\kappa$ (degree) \\
\hline \multirow{3}{*}{0561} & $\mathrm{~F}$ & -0.0099 & -0.0324 & 0.0137 \\
\cline { 2 - 5 } & $\mathrm{N}$ & -0.0062 & 0.0001 & -0.0008 \\
\cline { 2 - 5 } & $\mathrm{B}$ & 0.0163 & 0.0320 & 0.0057 \\
\hline \multirow{3}{*}{0562} & $\mathrm{~F}$ & -0.0532 & -0.0311 & 0.0332 \\
\cline { 2 - 5 } & $\mathrm{N}$ & -0.0254 & 0.0119 & 0.0046 \\
\cline { 2 - 5 } & $\mathrm{B}$ & 0.0149 & 0.0432 & 0.0090 \\
\hline 0563 & $\mathrm{~F}$ & -0.0162 & -0.0416 & 0.0183 \\
\hline
\end{tabular}

\begin{tabular}{|c|c|c|c|c|}
\hline & $\mathrm{N}$ & -0.0035 & 0.0022 & -0.0004 \\
\cline { 2 - 5 } & $\mathrm{B}$ & 0.0202 & 0.039 & 0.0056 \\
\hline
\end{tabular}

Table 3. Correction values of attitude angle bias for CE-1 CCD images

\begin{tabular}{|c|c|c|c|c|c|}
\hline \multirow{2}{*}{$\begin{array}{c}\text { Orbit } \\
\text { No. }\end{array}$} & \multirow[b]{2}{*}{ Image } & \multicolumn{2}{|c|}{ Column direction } & \multicolumn{2}{|c|}{ Row direction } \\
\hline & & $\begin{array}{c}\text { Mean } \\
\text { (pixel) }\end{array}$ & $\begin{array}{c}\text { RMS } \\
\text { (pixel) }\end{array}$ & $\begin{array}{c}\text { Mean } \\
\text { (pixel) }\end{array}$ & $\begin{array}{c}\text { RMS } \\
\text { (pixel) }\end{array}$ \\
\hline \multirow{3}{*}{0561} & $\mathrm{~F}$ & 0.00 & 0.12 & 0.00 & 0.00 \\
\hline & $\mathrm{N}$ & -0.00 & 0.20 & 0.00 & 0.00 \\
\hline & B & 0.00 & 0.19 & 0.00 & 0.00 \\
\hline \multirow{3}{*}{0562} & $\mathrm{~F}$ & -0.00 & 0.13 & 0.00 & 0.00 \\
\hline & $\mathrm{N}$ & -0.00 & 0.21 & 0.00 & 0.00 \\
\hline & B & -0.00 & 0.16 & 0.00 & 0.00 \\
\hline \multirow{3}{*}{0563} & $\mathrm{~F}$ & 0.00 & 0.16 & 0.00 & 0.00 \\
\hline & $\mathrm{N}$ & 0.00 & 0.26 & 0.00 & 0.00 \\
\hline & B & 0.00 & 0.18 & 0.00 & 0.00 \\
\hline
\end{tabular}

Table 4. Back-projection residuals for CE-1 CCD images after attitude angle bias correction

Figure 4 shows us that the residuals distributed in column direction before and after adjustment (correction of attitude angle bias) for forward-, nadir-, backward-looking images of No.0562 orbit.

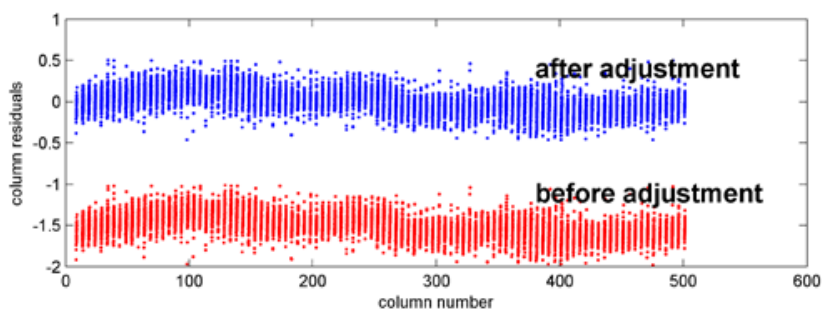

(a) Residuals of forward-looking image

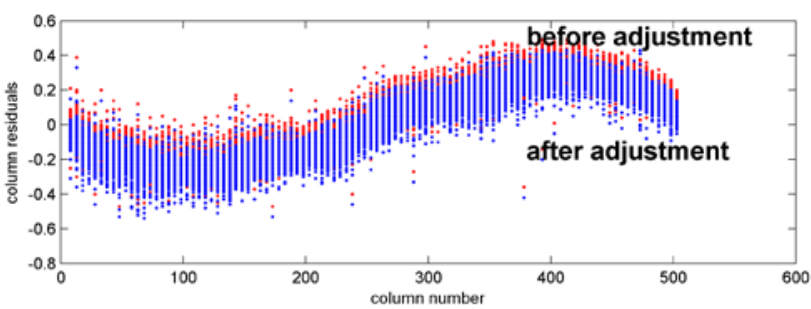

(b) Residuals of nadir-looking image

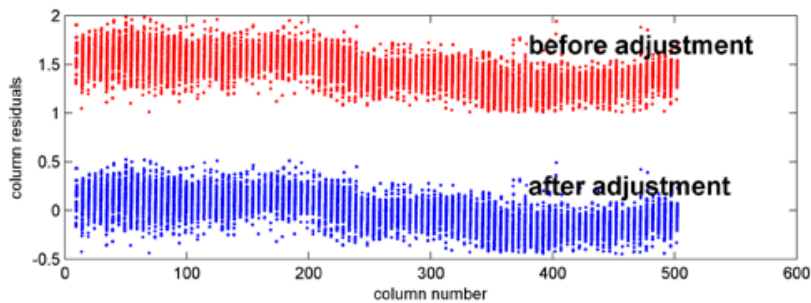

(c) Residuals of backward-looking image

Figure 4. The residuals distributions in column direction before and after adjustment (take No.0562 orbit for example)

It can be easily observed that after attitude angle bias correction, mean back-projection residuals of CE-1 CCD images are reduced to better than 1/100 pixel. After this refinement, DEM and DOM generated are automatically generated using CE-1 images and are shown in Figure 5. 


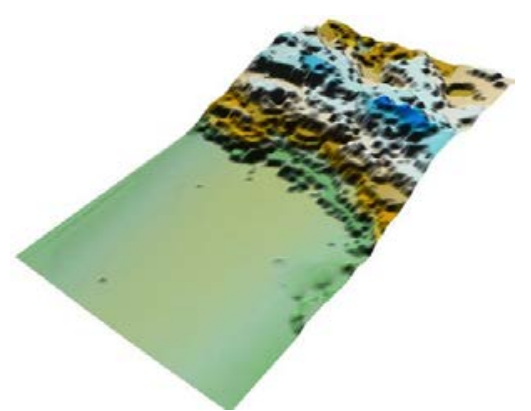

(a) DEM generated from CE-1 CCD images

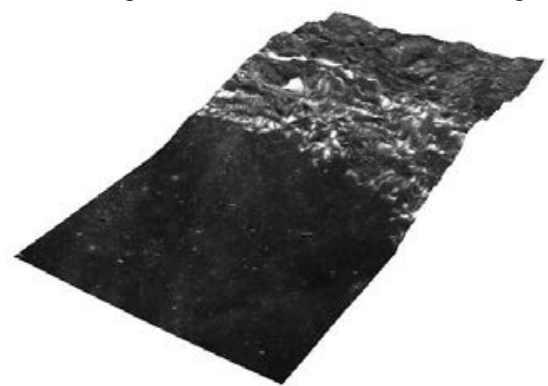

(b) DOM generated from CE-1 CCD images Figure 5. Partial DEM and DOM generated using CE-1 CCD images (take No.0562 orbit for example)

\subsection{Experiment on CE-2 CCD Images}

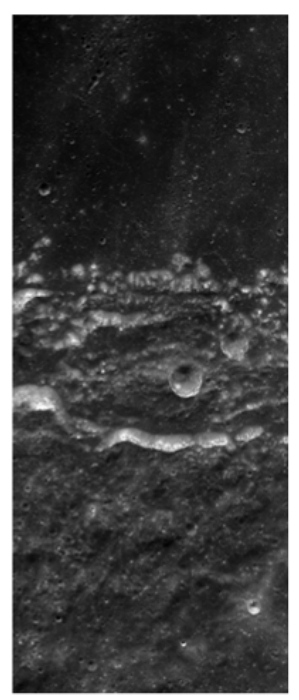

(a) Forward-looking

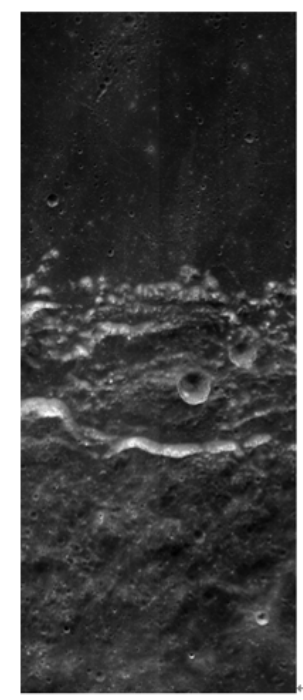

(b) Backward-looking
Figure 6. Partial forward- and backward-looking lunar images taken by CE-2 CCD camera from No.0581 orbit (7m resolution)

By now, all the images of CE-2 CCD camera with a resolution of $7 \mathrm{~m}$ can be applied from China Lunar Exploration and Aerospace Engineering Center. We also choose 3 orbits (No.0579, No.0580 and No.0581) of CE-2 CCD images located at Sinus Iridum. Figure 6 shows partial forward- and backward-looking images (15,000 rows, after grayscale stretching) of No. 0581 orbit.

In our experiment, we use 60,000 rows by 6,144 columns forward- and backward-looking images for each orbit. After feature point extraction, matching and gross error elimination (using RANSAC procedure), around 60,000 evenly distributed conjugate points for each stereo pair are obtained and used for space intersection and back-projection. Table 5 shows the re- siduals between the feature points and the back-projected points correspondently in image space which indicate that the residuals mainly exist in the column direction.

\begin{tabular}{|c|c|c|c|c|c|}
\hline \multirow{2}{*}{$\begin{array}{c}\text { Orbit } \\
\text { No. }\end{array}$} & \multirow{2}{*}{ Image } & \multicolumn{2}{|c|}{ Column direction } & \multicolumn{2}{c|}{ Row direction } \\
\cline { 3 - 6 } & & $\begin{array}{c}\text { Mean } \\
\text { (pixel) }\end{array}$ & $\begin{array}{c}\text { RMS } \\
\text { (pixel) }\end{array}$ & $\begin{array}{c}\text { Mean } \\
\text { (pixel) }\end{array}$ & $\begin{array}{c}\text { RMS } \\
\text { (pixel) }\end{array}$ \\
\hline \multirow{2}{*}{0579} & $\mathrm{~F}$ & -22.77 & 2.22 & 0.00 & 0.00 \\
\cline { 2 - 6 } & $\mathrm{B}$ & 22.73 & 2.21 & 0.00 & 0.00 \\
\hline \multirow{2}{*}{0580} & $\mathrm{~F}$ & -23.46 & 1.93 & 0.00 & 0.00 \\
\cline { 2 - 6 } & $\mathrm{B}$ & 23.42 & 1.92 & 0.00 & 0.00 \\
\hline \multirow{2}{*}{0581} & $\mathrm{~F}$ & -22.34 & 1.81 & 0.00 & 0.00 \\
\cline { 2 - 6 } & $\mathrm{B}$ & 22.30 & 1.81 & 0.00 & 0.00 \\
\hline
\end{tabular}

Table 5. Back-projection residuals in image space for CE-2 CCD images before adjustment

Comparing with Table 2, it is obvious that the back-projection residuals of CE-2 images are much larger than those of CE-1 images. As analyzed early, this is mainly because the two linear arrays of the CE-2 camera are separately assembled on the focal plane, while the three line CE-1 camera is realized by one area array sensor. Thus, the large residuals in column direction are not mainly caused by attitude angle bias but by errors of interior orientation model. We fitted LSLs of column residuals versus column position for forward- and backward-looking images of these three orbits. The coefficients $a$ and $b$ of them are listed in Table 6.

\begin{tabular}{|c|c|c|c|c|}
\hline \multirow{2}{*}{$\begin{array}{c}\text { Orbit } \\
\text { No. }\end{array}$} & \multicolumn{2}{|c|}{ Coefficient $a$} & \multicolumn{2}{c|}{ Coefficient $b$} \\
\cline { 2 - 5 } & F & B & F & B \\
\hline 0579 & -0.0011020 & 0.0011000 & -23.02 & 22.97 \\
\hline 0580 & -0.0008601 & 0.0008583 & -23.46 & 23.42 \\
\hline 0581 & -0.0009592 & 0.0009575 & -22.19 & 22.15 \\
\hline
\end{tabular}

Table 6. Coefficients $a$ and $b$ of the fitted lines of column residuals for forward- and backward-looking images of CE-2

Mean values of coefficients $a$ and $b$ of three orbits in Table 6 are converted to scale and offset and then used in Equation (8) to refine the interior orientation model. Back-projection residuals are calculated again after the refinement. Table 7 shows the results which indicate that residuals in column direction are reduced significantly from over 20 pixels to 0.02 pixel .

\begin{tabular}{|c|c|c|c|c|c|}
\hline \multirow{2}{*}{$\begin{array}{c}\text { Orbit } \\
\text { No. }\end{array}$} & \multirow{2}{*}{ Image } & \multicolumn{2}{|c|}{ Column direction } & \multicolumn{2}{c|}{ Row direction } \\
\cline { 3 - 6 } & & $\begin{array}{c}\text { Mean } \\
\text { (pixel) }\end{array}$ & $\begin{array}{c}\text { RMS } \\
\text { (pixel) }\end{array}$ & $\begin{array}{c}\text { Mean } \\
\text { (pixel) }\end{array}$ & $\begin{array}{c}\text { RMS } \\
\text { (pixel) }\end{array}$ \\
\hline \multirow{2}{*}{0579} & $\mathrm{~F}$ & -0.02 & 1.22 & 0.00 & 0.00 \\
\cline { 2 - 6 } & $\mathrm{B}$ & 0.02 & 1.22 & 0.00 & 0.00 \\
\hline \multirow{2}{*}{0580} & $\mathrm{~F}$ & -0.02 & 1.22 & 0.00 & 0.00 \\
\cline { 2 - 6 } & $\mathrm{B}$ & 0.02 & 1.22 & 0.00 & 0.00 \\
\hline \multirow{2}{*}{0581} & $\mathrm{~F}$ & -0.02 & 0.81 & 0.00 & 0.00 \\
\cline { 2 - 6 } & $\mathrm{B}$ & 0.02 & 0.80 & 0.00 & 0.00 \\
\hline
\end{tabular}

Table 7. Back-projection residuals in image space for CE-2 CCD images after interior orientation model refinement

The residuals distributed in column direction before and after adjustment (interior orientation model refinement) as well as the LSLs are shown in Figure 7 which visually demonstrated the effectiveness of this method.

We can also observe that the RMS residuals are relatively larger than the mean residuals, e.g., up to 1.22 pixels. This indicates that there is still room for further improvement, for example, fitting a $2^{\text {nd }}$ order polynomial may generate better result than fit- 
ting a straight line.

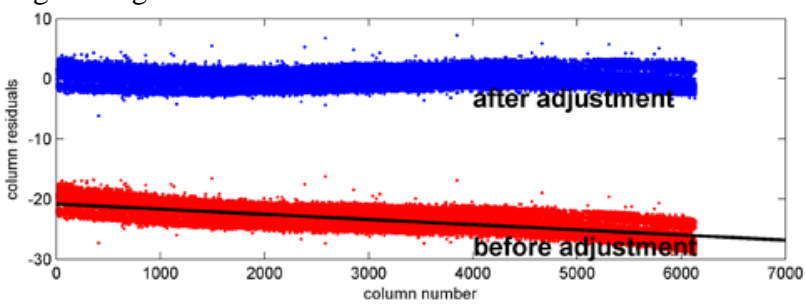

(a) Residuals and LSL for forward-looking image

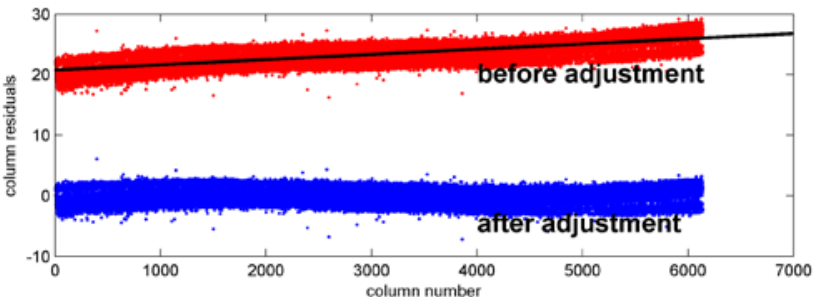

(b) Residuals and LSL for backward-looking image

Figure 7. Back-projection residuals in column direction before and after adjustment as well as the LQL (Take No.0580 orbit for example)

DEM and DOM are generated using the corrected interior orientation model and shown in Figure 8.

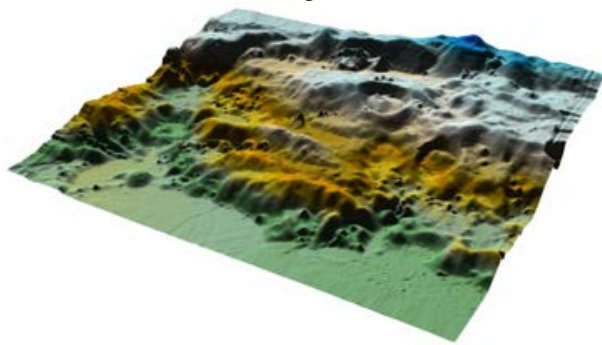

(a) DEM generated from CE-2 CCD images

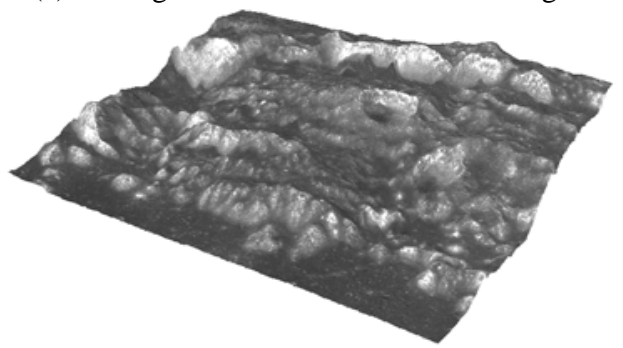

(b) DOM generated from CE-2 CCD images

Figure 8. Partial DEM and DOM generated using CE-1 CCD images (take No.0581 orbit for example)

\section{CONCLUSION AND DISCUSSION}

In this research, we have developed the rigorous sensor models of CE-1 and CE-2 CCD cameras to calculate ground points in object space and back-project ground points into image space. By analyzing back-projection residuals, we proposed two methods to refine the sensor models so that to reduce the residuals. Experimental results indicate that the two methods, correcting attitude angle bias and refining interior orientation model, are very effective for CE-1 and CE-2 images respectively. Consequently, the mapping products DEM and DOM have better precision after the sensor model refinement.

In the future, the two sensor model refinement methods will be further improved and tested using more and larger images from different orbits. Furthermore, a combined bundle adjustment incorporating these refinement methods will be developed to reach best precisions of CE- 1 and CE- 2 images.

\section{ACKNOWLEDGEMENTS}

Funding of this research by National Key Basic Research and Development Program of China (2012CB719902) and National Natural Science Foundation of China (40871202, 41171355) is acknowledged. We thank the Lunar and Deep Space Exploration Science Applications Center of the National Astronomical Observatories (NAOC) and Beijing Aerospace Control Center for providing the CE-1 and CE-2 images and telemetry data.

\section{REFERENCES}

Di. K., Yue. Z., Peng. M., et al., 2010. Co-registration of CHANG'E-1 stereo images and laser altimeter data for 3D mapping of lunar surface. ASPRS/CaGIS 2010 Specialty Conference, Orlando, Florida, USA, December 15-19.

Li, C., Liu, J., Ren, X., et al., 2010. The global image of the Moon obtained by the Chang'E- 1 Data processing and lunar cartography. Sci China Earth Sci, 40(3), pp.294-306.

Liu, Y., Di. K., 2011. Evaluation of rational function model for geometric modeling of Chang'E-1 CCD images. In: ISPRS Workshop, Geospatial Data Infrastructure: form Data Acquisition and Updating to Smarter Services, Guilin, China, pp. 121125 (CD-ROM).

Lowe, D.G., 1999. Object recognition from local scale-invariant features, Proc. of the 1999 International Conference on Computer Vision, 20-25 September, Corfu, Greece, pp. 1150-1157.

National Astronomical Observatories, Chinese Academy of Science (NAOC), 2010. Release of the first Chang'E-2 image of Sinus Iridum.

http://moon.bao.ac.cn/templates/T_yestem_articelcontent/index. aspx?nodeid=13\&page=ContentPage\&contentid=184 (last accessed 29 Mar. 2012)

Peng, M., Yue, Z., Liu, Y., et al., 2010. Research on lunar and Mars orbital stereo image mapping. Proc. of SPIE Remote Sensing of Environment: The 17th Remote Sensing Conference of China, 27-31 August, Hangzhou, China.

Wikipedia 2012 . Chinese Lunar Exploration Program, http://en.wikipedia.org/wiki/Chinese_Lunar_Exploration_Progr am (29 Mar. 2012)

Xinhua News Agency, 2012. China released a high resolution global map of the Moon. Xinhua net, http://news.xinhuanet.com/politics/201202/06/c_111491784_3.htm?prolongation=1 (last accessed 29 Mar. 2012)

Yuan, X., Yu, J., 2008. Calibration of Constant Angular Error for High Resolution Remotely Sensed Imagery. Acta Geodaetica Et Acrtographica Sinica, 37(1), pp. 36-41.

Zhao, B., Wen, D., Yang, J., 2011a. Two bore-sight stereo mapping with single lens, TDI CCD pushing model imaging and compensations of the speed-to-height rate - Chang'e-2 CCD Camera. Acta Optica Sinica, 31(9), pp. 126-133.

Zhao, B., Yang, J., Wen, D., 2011b. Chang'e-2 satellite CCD stereo camera design and verification. Spacecraft Engineering, 20(1), pp. 14-21. 